\title{
De Alma-Ata a Astana: o percurso dos Cuidados de Saúde Primários em Portugal,1978-2018 e a génese da Medicina Familiar
}

\author{
From Alma-Ata to Astana: the path of Primary Health Care in \\ Portugal, 1978-2018 and the genesis of Family Medicine
}

Luis Pisco (https://orcid.org/0000-0002-9007-8949) ${ }^{1}$

Luiz Felipe Pinto (https://orcid.org/0000-0002-9888-606X) ${ }^{2}$

${ }^{1}$ Administração Regional de Saúde de Lisboa e Vale do Tejo. Av. Estados Unidos da América 77, 1700-179

Lisboa Portugal.luis.pisco@ arslvt.min-saude.pt

${ }^{2}$ Faculdade de Medicina, Universidade Federal do Rio de Janeiro. Rio de Janeiro RJ Brasil.

\begin{abstract}
Throughout the twentieth century, the profound changes that have taken place in $\mathrm{Me}$ dicine can only be wholly explained if observed from a historical perspective, for they have always occurred in response to external influences, some scientific and technological, others of a social nature. Modern Family Medicine is one of the many new disciplines that have developed during medical history, and we critically discuss the last 40 years of primary health care in Portugal, which started in 1971, long before the Alma-Ata Declaration (1978). Along the way, in 2005, the Primary $\mathrm{He}$ alth Care Reform emerges in Portugal, along with the new family health facilities, which until September 2019, attended about $94 \%$ of Portuguese citizens, i.e., 9,5 million people. At the end of this course, in solidarity and voluntarily, this Reform inspired another one in Brazil, in Rio de Janeiro, in 2009. Finally, we present the challenges pointed out in the 2018 Astana Declaration, among them, the issue of the workforce in primary health care as an essential factor for the performance and sustainability of health systems.
\end{abstract}

Key words Primary health care, Family Medicine, Universal health coverage
Resumo Ao longo século XX, as profundas alterações que ocorreram na Medicina apenas podem ser completamente esclarecidas se forem observadas numa perspectiva histórica, pois elas sempre ocorreram em resposta a influências externas, umas científicas e tecnológicas, outras de ordem social. A moderna Medicina Familiar é uma das muitas disciplinas novas que se desenvolveram durante o curso da história da Medicina e aqui debatemos de forma crítica, os últimos 40 anos dos cuidados primários em saúde em Portugal, começando em 1971, mesmo antes da Declaração de Alma-Ata (1978). Ao longo do percurso, em 2005, surge a Reforma dos Cuidados Primários em Saúde em Portugal e as novas unidades de saúde familiar, que até setembro de 2019 atendiam cerca de $94 \%$ dos cidadãos portugueses, ou seja, mais de nove milhões e meio de pessoas. No final dessa trajetória, de forma solidária e voluntária, esta Reforma serviu de inspiração para outra, no Brasil, na cidade do Rio de Janeiro, em 2009. Por fim, apresentamos os desafios apontados na Declaração de Astana de 2018, dentre elas, a questão da força de trabalho nos cuidados de saúde primários, como fator essencial para o desempenho e a sustentabilidade dos sistemas de saúde.

Palavras-chave Cuidados primários em saúde, Medicina familiar, Cobertura universal de saúde 


\section{Introdução}

Na sua obra "A textbook of Family Medicine", McWhinney, considerado o pai da Medicina Familiar do Canadá1,2, refere que as profundas alterações que ocorreram na Medicina apenas podem ser completamente esclarecidas se forem observadas numa perspectiva histórica, pois elas sempre surgiram em resposta a influências exteriores, umas científicas e tecnológicas, outras de ordem social. A moderna Medicina Familiar é uma das muitas disciplinas novas que se desenvolveram durante o curso da história da Medicina.

Em 1910, o Relatório Flexner ${ }^{3}$ descrevia grandes deficiências no sistema de ensino das faculdades de Medicina dos Estados Unidos, e conduziu mesmo ao encerramento de muitas delas por falta de credibilidade científica. O impacto daquele relatório teve repercussões não só nos Estados Unidos como também na Europa. O rigor de critérios e requisitos que passou a ser exigido às escolas médicas transformou-as em centros de ensino e investigação. Criou, ainda, as condições propícias para o desenvolvimento de uma medicina tecnológica, altamente especializada, embora menos sensível ao papel social da profissão médica.

No Reino Unido, surgiu pela primeira vez, no chamado Relatório Dawson de $1920^{4}$, um posicionamento do governo britânico, que por um lado procurava contrapor-se ao modelo de Flexner, de cunho curativo, fundado no reducionismo biológico, enquanto que por outro buscava constituir-se uma referência para a organização do modelo de cuidados de saúde britânico, marcado, já naquela altura, por elevados custos, complexidade médica e baixa resolução dos problemas dos cidadãos.

O Relatório Dawson preconizava a organização dos serviços em centros de saúde e hospitais, serviços domiciliários, serviços complementares e hospitais universitários. Os centros de saúde e os serviços domiciliários deveriam organizar-se de acordo com um modelo regional em que a maior parte dos problemas de saúde deveriam ser resolvidos por médicos com formação em Medicina Geral e Familiar. Só os casos que o médico de família não tivesse condições de solucionar deveriam ser encaminhados para os hospitais.

Esta organização, caracterizada pela hierarquização dos níveis de cuidados e pela integralidade, ou seja, o fortalecimento da relação entre acções curativas e preventivas, influenciou a organização dos sistemas de saúde de todo o mundo.
Ao longo das três décadas seguintes, o rápido progresso tecnológico, o desenvolvimento da investigação aplicada e a crescente especialização, fonte de prestígio no exercício médico, não favoreceram a prática da Clínica Geral. Depois da II Guerra Mundial, o lugar da Clínica Geral foi ocupado pela intervenção médica decorrente da fragmentação das grandes especialidades em sub-especialidades. Até no Reino Unido, onde os clínicos gerais mantiveram uma posição importante no sistema de saúde, reforçada após a criação do National Health Service (NHS), em 1948, se verificou esse declínio.

A resposta dos clínicos gerais manifesta-se com a criação de academias e colégios destinados a impulsionar a prática da medicina familiar. Nomeadamente, a criação da American Academy of Family Physicians, nos Estados Unidos da América, em 1947, do Royal College of General Practitioners, no Reino Unido, em 1952, e do College of Family Physicians, no Canadá, em 1954.

A questão é que o processo de ultra-especialização do exercício médico moderno gerou uma tendência para a despersonalização da relação médico-doente que conduz à perda da percepção integral da pessoa humana, agora dividida em sistemas, aparelhos e órgãos, ao mesmo tempo que relegava para segundo plano, ou ignorava, a dimensão psicossocial do indivíduo.

Todos estes elementos tiveram uma influência decisiva no processo de consciencialização social relativamente à necessidade de resgatar $\mathrm{o}$ velho clínico geral, o médico que acompanhava a história da pessoa e da família, desde o nascimento até à morte.

Quando o domínio da especialização parecia ter atingido o auge, surge nos Estados Unidos um movimento contrário à fragmentação de cuidados, que defende o reaparecimento do clínico geral, agora diferente do "velho clínico geral", mediante um papel bem diferenciado no que diz respeito à sua actuação como profissional médico. Este movimento é iniciado, segundo McWhinney ${ }^{1}$, com dois documentos essenciais: "The Graduate Education of Physicians: Report of the Citizens Committee on Graduate Medical Education" $\mathrm{e}$ "Meeting the Challenge of Family Practice: Report of the Ad Hoc Committee on Education for Family Practice of the Council on Medical Education"6.

Enquanto o primeiro - o Relatório da Comissão Millis - desacreditava a fragmentação nos cuidados de saúde e afirmava it is time for a revolution, not a few patchwork adaptations, o segundo - o Relatório da Comissão Willard - defendia 
a formação e o treino de um novo especialista: o médico de família ${ }^{5,6}$.

Três anos mais tarde, o American Board of Family Practice dá origem à "20th Medical Speciality Board", com o reconhecimento formal da Medicina Familiar como especialidade médica. Curiosamente, a primeira com a exigência de recertificação de seis em seis anos. Este desenvolvimento foi de tal modo vigoroso nos Estados Unidos que, em 1971, a própria Academia Americana de Clínica Geral mudou a designação para Academia Americana de Medicina Familiar.

A partir da década de 1970, movimentos similares, embora de diferentes dimensões e com particularidades próprias, ocorreram paralelamente no Canadá, Reino Unido, Holanda, Noruega, Austrália, Portugal, Espanha e outros países desenvolvidos. E, na sequência deste movimento internacional, é criada a World Organization of Family Doctors (WONCA), cuja primeira conferência tem lugar, em 1972, em Melbourne. Seis anos mais tarde, ocorre um acontecimento ainda mais decisivo: a Conferência de Alma-Ata.

No século XX, a história da Medicina Familiar passou a estar intrinsecamente ligada à história dos Cuidados de Saúde Primários (CSP), entendidos como o primeiro nível de cuidados do sistema de saúde.

\section{Conferência de Alma-Ata}

Nesse contexto, em 1978, a Organização Mundial da Saúde (OMS) e o Fundo das Nações Unidas para a Infância (UNICEF) realizam a I Conferência Internacional sobre Cuidados de Saúde Primários, em Alma-Ata, no Cazaquistão (antiga União Soviética). Nesta conferência, a OMS propõe atingir a meta "Saúde para todos no ano 2000", através da implementação e desenvolvimento, em todo o mundo, dos "Cuidados de Saúde Primários”. Estes são definidos como cuidados essenciais de saúde, baseados em métodos e tecnologias práticas, cientificamente bem fundamentadas e socialmente aceitáveis, colocados ao alcance universal de indivíduos e famílias da comunidade, mediante a sua plena participação e a um custo que a comunidade e o país possam manter em cada fase do seu desenvolvimento, num espírito de auto-confiança e auto-determinação. Fazem parte integrante, quer do sistema de saúde do país, do qual constituem a função central e o foco principal, quer do desenvolvimento social e económico da sociedade. Representam o primeiro nível de contacto dos indivíduos, da família e da comunidade com o sistema nacional de saúde, pelo qual os cuida- dos de saúde são levados o mais próximo possível dos locais onde as pessoas habitam e trabalham, $e$ constituem o primeiro elemento de um continuado processo de assistência à saúde $e^{7}$.

A Declaração de Alma-Ata, como foi chamado o pacto assinado entre os 134 países membros da OMS, propõe a instituição de serviços locais de saúde centrados nas necessidades da população e fundados numa perspectiva interdisciplinar, com o envolvimento de médicos, enfermeiros, parteiras, auxiliares e agentes comunitários, bem como com a participação social na gestão e no controlo das suas actividades.

O documento descreve ainda as acções mínimas necessárias para o desenvolvimento dos cuidados de saúde primários nos diversos países: educação para a saúde, voltada para a prevenção e protecção; distribuição de alimentos e nutrição apropriada; tratamento da água e saneamento; saúde materno-infantil; planeamento familiar; vacinação; prevenção e controlo de doenças endémicas; tratamento de doenças e lesões comuns; e, por último, fornecimento de medicamentos essenciais.

Mais do que isso, a Declaração de Alma-Ata aponta para a necessidade de sistemas de saúde universais, isto é, concebe a saúde como um direito humano. Apesar das metas de Alma-Ata não terem sido alcançadas plenamente, tornaram-se uma referência fundamental para as reformas ocorridas nos sistemas de saúde de diversos países nos anos 1980 e 1990 do século XX, incluindo Portugal, que como veremos adiante, iniciou esse processo de mudanças ainda na década de 1970 .

\section{O prenúncio dos primeiros centros de saúde em Portugal no percurso dos cuidados de saúde primários}

Em 1971, a Reforma do Sistema de Saúde e da Assistência (Decreto-Lei $\left.n^{\circ} 413 / 71\right)^{8}$ conduziu à reorganização dos serviços do Ministério da Saúde (criado em 1958), com os chamados "centros de saúde de primeira geração". Este Decreto introduziu mudanças significativas no sistema, ao reestruturar os serviços centrais e regionais e de âmbito local. Tentava-se, pela primeira vez, contrariar o domínio dos hospitais e orientar a prestação de cuidados para os cuidados primários. Além disso, estes centros de saúde foram dotados de múltiplas valências, incluindo, naturalmente, a prestação de cuidados médicos elementares e de enfermagem. Essencialmente, o seu objectivo era proporcionar alguma protecção à população (com especial ênfase em dois grupos particular- 
mente vulneráveis, as mulheres e as crianças) e prevenir as principais doenças infecto-contagiosas através da vacinação.

A Revolução de Abril de 1974 originou profundas alterações no país e nas instituições do Estado, incluindo a Saúde. O fim da guerra no Ultramar e a independência das colónias implicou o regresso de milhares de portugueses num curto espaço de tempo. Muitos médicos saídos das faculdades e que, anteriormente, seriam obrigados a ir para o Ultramar, ficaram no país. Muitos deles escolheram a Clínica Geral. Isso permitiu, em 1975, a criação do "serviço médico à periferia", que levou os médicos recém-licenciados para fora dos grandes centros urbanos e criou a oportunidade de melhorar a assistência médica nos centros de saúde, incluindo as zonas rurais e o Interior.

\section{O nascimento do Serviço Nacional de Saúde (SNS) em Portugal}

A criação do Serviço Nacional de Saúde (SNS) ocorre alguns anos depois, em 1979, com António Arnaut, conhecido como o "pai" do SNS. Financiado e assegurado pelo Estado, o SNS foi concebido como um serviço universal, geral e gratuito, independentemente das capacidades económicas dos cidadãos. De facto, o artigo 64 da Constituição portuguesa de 1976 determina, em primeiro lugar, que todos têm direito à protecção da saúde e o dever de a promover e a defender'. Em segundo termo, refere que o direito à protecção da saúde é realizado pela criação de um Serviço $\mathrm{Na}$ cional de Saúde, universal, geral e gratuito (... $)^{9}$.

Neste contexto, a implementação da Clínica Geral como especialidade médica, iniciada nos anos 1970, experimenta um forte desenvolvimento com a criação, entre 1981 e 1983, dos Institutos de Clínica Geral, no Norte, Centro e Sul, dada a relevância dos clínicos gerais no SNS. Uma das prioridades destes Institutos - que se encontravam sob a tutela da Direcção-Geral dos Cuidados de Saúde Primários (criada pelo Decreto -Lei no $74-\mathrm{C} / 84$, de 2 de Março ${ }^{10}$ - foi a formação dos médicos dos cuidados de saúde primários, segundo dois modelos distintos: o Internato Complementar de Clínica Geral e a Formação Específica em Exercício. Este último modelo constituiu uma experiência inovadora analisada, anos mais tarde, por outros países, que a adaptaram à sua própria realidade - uma vez que se destinava a médicos que já estavam a exer- cer a profissão de Clínicos Gerais nos centros de saúde. Integrava o "exercício orientado", a "formação em sala", ou seja, em moldes tradicionais, e o estágio hospitalar. Através deste modelo de formação em serviço, os Clínicos Gerais tiveram a possibilidade de obter o grau de assistente de clínica geral e, consequentemente, a entrada na carreira de Clínica Geral.

Em 1982 surge a carreira de Clínica Geral (Diploma das Carreiras Médicas, Decreto-Lei no $310 / 82)^{11}$ e, no ano seguinte, são criados os chamados "centros de saúde de segunda geração" (Despacho normativo no 97/83, de 22 de Abril) ${ }^{12}$. Estes centros de saúde resultam da fusão dos Postos da Caixa de Previdência (criados pelo Estado Novo) com os centros de saúde de primeira geração.

O Colégio de Clínica Geral da Ordem dos Médicos é criado nesse mesmo ano. Em 1983, surge a Associação Portuguesa de Médicos de Clínica Geral (APMCG) que, nos anos seguintes, se transformaria na maior associação profissional médica de inscrição não obrigatória.

Na década de 1980 , a gestão do SNS e a necessidade de conter os custos crescentes com a Saúde começou a constituir uma prioridade para os governos. A revisão da Constituição, em 1989, torna a saúde "tendencialmente gratuita" e, um ano mais tarde, é aprovada a Lei de Bases da Saúde ${ }^{13,14}$. Genericamente, este importante documento refere que a saúde passa a ser responsabilidade não só do Estado mas de cada indivíduo e permite o aumento da contratação de serviços do SNS com o sector social e privado. Nesse mesmo ano, são introduzidas, pela primeira vez, taxas moderadoras nos centros de saúde e hospitais. O pagamento dessas taxas moderadoras ou a comparticipação isentavam pessoas em situações especiais, como aquelas com situações clínicas relevantes de maior risco de saúde ou situações de insuficiência económica.

A organização dos cuidados de saúde primários em torno dos chamados centros de saúde de segunda geração, sem autonomia administrativa e financeira, na prática manteve-os na dependência das sub-regiões de Saúde (SRS) e das Administrações Regionais de Saúde (ARS). A necessidade de alterar este estado de coisas e de aproximar o centro de decisão do terreno e dos profissionais fez com que, em 1996, começassem a surgir uma série de experiências organizacionais, lideradas por médicos de família pertencentes à APMCG. 
As décadas de 1990 e meados dos anos 2000: os Projectos Alfa e a criação dos centros de saúde de terceira geração

Em 1996 e 1997 surgem, na Região de Lisboa e Vale do Tejo, os Projectos Alfa ${ }^{15}$. Essencialmente, visavam estimular soluções organizativas, a partir da iniciativa dos próprios profissionais de saúde, que permitissem aproveitar melhor a capacidade e os meios existentes nos centros de saúde. O modelo estava assentado em dois princípios fundamentais: por um lado, a melhoria da acessibilidade e da qualidade dos cuidados de saúde primários e, por outro, a constituição de pequenas equipas multiprofissionais, responsáveis pela prestação de cuidados de saúde a uma determinada população, de acordo com princípios, objectivos e metas bem definidos e acordados com a Administração, através de um processo de contratualização.

A avaliação positiva destes projectos conduziu à aprovação, em 1998, do Regime Remuneratório Experimental (RRE) para os médicos de Clínica Geral. O RRE foi enquadrado pelo Decreto-Lei $\mathrm{n}^{\circ} 117 / 98$, de 5 de $\mathrm{Maio}^{16}$, que consagrava não só alterações na organização do trabalho semelhantes às dos Projectos Alfa, como introduzia uma modalidade de remuneração dos médicos associada à quantidade de trabalho desenvolvido e à qualidade profissional. $\mathrm{O}$ modelo, inovador, agitou uma Administração Central burocrática e imobilista. Pela primeira vez, o bom desempenho clínico era alvo de discriminação positiva e a criação das Agências de Contratualização, em 1997 e 1999, vieram contribuir para a separação entre o financiamento e a prestação de cuidados de saúde. Em 1998 iniciam-se as discussões dos contratos-programa com os hospitais e, mais tarde, sucede o mesmo com os centros de saúde.

O debate sobre a descentralização da gestão dos cuidados de saúde primários das sub-regiões de Saúde (SRS) para os centros de saúde culmina, em 1999, com a legislação relativa aos centros de saúde de terceira geração (Decreto-Lei no 157/99, de 10 de maio $)^{17}$. Estas unidades de saúde, com personalidade jurídica, autonomia administrativa e financeira, deveriam organizar-se em unidades funcionais, com autonomia técnica, ligadas entre si, de acordo com um modelo de trabalho multiprofissional e em rede. O objectivo era flexibilizar a gestão, aproximando-a dos profissionais que estavam no terreno e que, por isso mesmo, conheciam melhor as necessidades da população, como potenciar a proximidade com os utentes e melhorar o acesso. Contudo, os centros de saúde de terceira geração, apesar de todo o esforço e trabalho desenvolvido durante anos, não chegaram a sair do papel. Em grande medida, devido à resistência das sub-regiões de Saúde, que veriam o seu poder transferido para os centros de saúde, assim que estes tivessem autonomia administrativa e financeira. A sua extinção estava pré-determinada. E, de facto, aconteceu.

\section{A segunda metade dos anos 2000 e a década de 2010: da Missão dos Cuidados Primários em Saúde à Reforma do Sistema de Saúde}

O Programa do XVII Governo Constitucional (2005-2009), na área da saúde, atribuiu um particular enfoque aos cuidados primários e à sua importância na ligação com os utentes, por ser o primeiro acesso deste aos cuidados de saúde. Referia explicitamente que os centros de saúde seriam reestruturados com a criação de novas unidades, as "unidades de saúde familiar" (USF) ${ }^{18}$. Em linhas gerais, essas novas unidades deveriam obedecer aos seguintes princípios: (i) pequenas equipas multiprofissionais e auto-organizadas; (ii) autonomia organizativa funcional e técnica; (iii) contratualização de uma carteira básica de serviços; (iv) meios de diagnósticos descentralizados; (v) sistema retributivo que considerasse a produtividade, a acessibilidade e a qualidade.

Na sequência do estabelecido no Programa do Governo, em setembro de 2005, o Conselho de Ministros ${ }^{19}$ criou, na dependência direta do então Ministro da Saúde Correia de Campos, a intitulada "Missão para os Cuidados de Saúde Primários" (MCSP), com a natureza de estrutura de missão, para a condução do projecto global da reforma dos cuidados primários em Portugal. A MCSP foi prorrogada por dois anos e posteriormente mais um ano, terminando o seu mandato em 14 de abril de 2010, após quatro anos e meio de actividade. Publicou, entre outros, um importante documento, intitulado "Linhas de Acção Prioritária para o Desenvolvimento dos Cuidados de Saúde Primários”20.

Estabeleceu como grandes objetivos para a Reforma da Atenção Primária a obtenção de mais e melhores cuidados de saúde para os cidadãos, aumento da acessibilidade e consequente crescimento da satisfação dos utilizadores dos serviços. Pretendeu-se também aumentar a satisfação dos profissionais, criando boas condições de trabalho, melhorando a organização e recompensando as boas práticas. Simultaneamente, planejou a melhoria da eficiência e promoveu a contenção de custos, eliminando concorrências estruturais, obtendo economias de escala. 
Ao longo da década de 2010, centenas de USF foram inauguradas em todo o País e dezenas de centros de saúde foram transformados em USF. Em 2019, mais de 9,5 milhões de cidadãos portugueses tinham médico de família atribuído (5.765 em todo o País), o que correspondia a cerca de $94 \%$ do total da população-residente. No caso de todos os médicos aceitarem as posições do concurso que prevê 400 vagas de medicina geral e familiar, a percentagem de portugueses com médico de família pode chegar aos $98 \%{ }^{21}$. É importante também destacar que nessa mesma década, em um longo processo de parcerias com o Brasil, o modelo dos cuidados de saúde primários serviram de fonte de inspiração no período de 20092016, para a Reforma da Atenção Primária à Saúde realizada na cidade do Rio de Janeiro ${ }^{22,23}$.

\section{A Declaração de Astana e os desafios para o futuro dos cuidados de saúde primários}

No segundo dia da Conferência em Astana, realizada entre 25 e 26 de outubro de 2018, a Revista The Lancet publicou uma edição especial dedicada aos cuidados de saúde primários ${ }^{24}$, destacando que os cuidados de saúde primários fortes, enraizados na participação e ação da comunidade, são a base de todo o sistema de saúde, e nenhum país pode alcançar saúde para todos sem eles.

E, se regressarmos a este Editorial do Lancet de outubro de $2018^{24}$, poderemos constatar que os cuidados de saúde primários estão em crise. Ou seja, há enormes desafios a serem enfrentados, pois no primeiro nível de atenção à saúde, estes cuidados estão subdesenvolvidos em muitos países, subfinanciados noutros e enfrentam uma grande missão de recrutamento e retenção da sua força de trabalho. Metade da população mundial não tem acesso aos serviços de saúde essenciais. No entanto, de 80 a $90 \%$ das necessidades de saúde das pessoas ao longo da sua vida poderiam ser prestadas dentro de uma estrutura básica de cuidados primários - desde cuidados de saúde materno-infantis, prevenção de doenças, vacinação, até a gestão de doenças crónicas e cuidados paliativos. À medida que as populações envelhecem e a multimorbilidade se torna a norma, o papel dos profissionais dos cuidados de saúde primários torna-se cada vez mais importante e, por isso, um renascimento dos cuidados de saúde primários é essencial para fornecer saúde para todos, sobretudo aos mais vulneráveis.

Investir em cuidados de saúde primários através de quatro plataformas (cuidados de base comunitária, centros de saúde, hospitais de pri- meiro nível e intervenções de base populacional) é uma das mensagens da Comissão Lancet sobre "Investir na Saúde"25.

A Declaração de Astana ${ }^{26}$ pode marcar o início de um futuro melhor para os cuidados de saúde primários, que como vimos em Portugal teve início em uma linha de tempo que começou em 1971 (Figura 1).

A força de trabalho em saúde (e por maioria de razões nos cuidados de saúde primários) é um fator essencial para o desempenho e a sustentabilidade dos sistemas de saúde. A Organização Mundial de Médicos de Família (WONCA) tem aumentado os seus esforços para formar médicos em países em desenvolvimento, mas nos últimos 30 anos houve investimento insuficiente em profissionais de cuidados de saúde primários. Equipas multiprofissionais focadas nas necessidades das pessoas podem ser uma maneira importante de introduzir inovações.

Portugal, felizmente, encontra-se a nível mundial entre os países com um sistema de saúde mais orientado para os cuidados de saúde primários, possuindo inclusive entre um de seus principais indicadores - a taxa de mortalidade infantil - uma acentuada queda nos últimos 40 anos (Figura 2).

Em Portugal o caminho de Alma-Ata a Astana é praticamente sobreponível ao percurso do Serviço Nacional de Saúde. Na celebração dos 40 anos, Pizarro $^{27}$ escreve que hoje estamos a par

\begin{tabular}{|c|c|}
\hline 1143 & Nação independente \\
\hline 1910 & Implantação da República \\
\hline 1971 & Centros de Saúde Comunitários \\
\hline 1974 & Regime Democrático \\
\hline 1978 & Conferência de Alma-Ata \\
\hline 1979 & Serviço Nacional de Saúde \\
\hline 1982 & Carreira de Clínica Geral \\
\hline 1986 & Membro da União Europeia \\
\hline 2005 & Reforma dos Cuidados de Saude Primários \\
\hline \multirow[t]{2}{*}{2009} & Reforma dos Cuidados Primários no Rio \\
\hline & de Janeiro \\
\hline 2018 & Conferência de Astana \\
\hline
\end{tabular}

Figura 1. Linha do tempo dos cuidados de saúde primários em Portugal: De Alma-Ata Astana, passando pelo Rio de Janeiro, Brasil. 


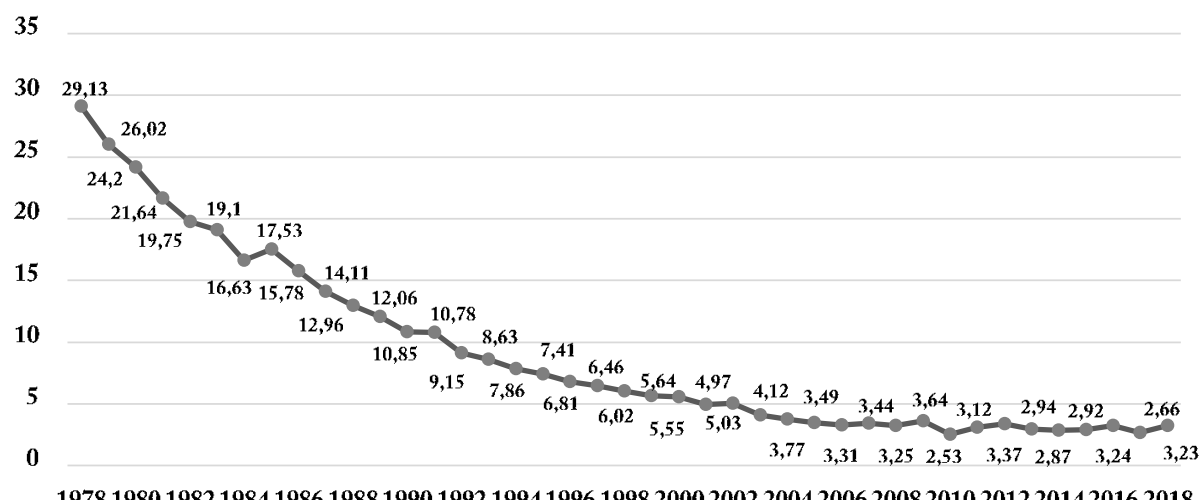

Figura 2. Evolução da taxa de mortalidade infantil - Portugal - 1978-2018.

Fonte: Instituto Nacional de Estatísticas (INE), Portugal.

dos países com melhores resultados na generalidade dos índices que avaliam os resultados em saúde. Temos resultados excelentes na mortalidade infantil e quase erradicamos a mortalidade materna. Tratamos as doenças cardiovasculares e o cancro ao nível dos melhores. Lideramos na complexa área da transplantação de órgãos. A esperança média de vida à nascença aumentou consideravelmente, situando-se agora acima dos 80 anos. Por isso, o SNS é sentido pela generalidade dos portugueses como uma das grandes conquistas da nossa democracia. Mas, para vencer os desafios do presente e para continuar a melhorar, não podemos deixar de lutar. Celebrar o SNS é, por isso, fazer o que ainda não foi feito.

Nesse mesmo sentido, por ocasião das celebrações oficiais dos 40 anos do SNS, a Ministra da Saúde, Marta Temido ${ }^{28}$, afirma que apesar da relevância do caminho percorrido e das suas conquistas, a forma mais importante de comemorar o $40^{\circ}$ aniversário do SNS será prepará-lo para o futuro. Foram identificadas três prioridades: o SNS precisa de melhorar a qualidade de acesso, motivar os profissionais de saúde para aumentar a sua produtividade e reforçar o investimento. $\mathrm{O}$ SNS é e deverá continuar a ser sobretudo um instrumento de combate às desigualdades e de reforço da coesão social.

Seria muito importante que a nova Lei de Bases da Saúde, promulgada em $2019^{29}$, pudesse trazer as melhorias que todos desejamos, e nos ajudasse a enfrentar os desafios atuais do Serviço
Nacional de Saúde, com grande solidariedade e cooperação entre todos os prestadores de serviços para benefício de todos os cidadãos portugueses.

\section{Colaboradores}

L Pisco e LF Pinto participaram, em conjunto, de todas as etapas de elaboração do artigo e revisão final do mesmo.

\section{Agradecimentos}

Agradecemos a todos os profissionais de saúde que nos últimos 40 anos do Serviço Nacional de Saúde (SNS) português integraram e integram ainda hoje, os cuidados de saúde primários. O autor LF Pinto, que no período de elaboração do artigo encontrava-se em estágio de pós-doutoramento no IHMT/UNL, gostaria de agradecer o apoio das sugestões e críticas recebidas ao longo da elaboração do texto. 


\section{Referências}

1. McWhinney IR. Manual de Medicina Familiar. Lisboa: Inforsalus; 1994.

2. Gusso GDF. O legado de Ian McWhinney. Rev Bras Med Fam Comunidade 2012; 7(24):204-206.

3. Flexner A. Medical Education in the United States and Canada: A Report to the Carnegie Foundation for the Advancement of Teaching. Washington: Science and Health Publications, Inc.; 1910.

4. Great Britain. Ministry of Health. Consultative Council on Medical and Allied Services. Interim Report. (Chairman Lord Dawson). London: HMSO; 1920.

5. Council on Medical Education and Hospitals of the American Medical Association, Citizens Commission on Graduate Medical Education. The Graduate Education of Physicians: The report of the Citizens Commission on Graduate Medical Education (Millis Report). Chicago: American Medical Association; 1966.

6. American Medical Association, Ad Hoc Committee on Education for Family Practice. Meeting the Challenge of Family Practice (the Willard report). Chicago: American Medical Association; 1966.

7. World Health Organization (WHO). Declaration of Alma-Ata: international conference on primary health care, Alma-Ata, USSR, 6-12 September 1978. Geneva: WHO; 1978.

8. Portugal. Decreto-Lei ${ }^{\circ}$ 413/71. Reforma do Sistema de Saúde e da Assistência. Diário da República 1971; 27 set.

9. Portugal. Constituição Portuguesa de 1976. Diário da República 1976; 2 abr.

10. Portugal. Decreto-Lei no $74-\mathrm{C} / 84$, de 2 de março de 1984. Diário da República 1984; 2 mar.

11. Portugal. Decreto-Lei no $310 / 82$. Diploma das Carreiras Médicas. Diário da República 1982; 3 ago.

12. Portugal. Despacho normativo no $97 / 83$, de 22 de abril de 1983. Criação dos centros de saúde de segunda geração. Diário da República 1983; 22 abr.

13. Portugal. Constituição de 1989. Diário da República 1989; 8 jul.

14. Portugal. Lei de Bases de saúde de 1990. Diário da República 1990; 24 ago.

15. Conceição C, Fronteira I, Hipólito F, Van Lerberghe W, Ferrinho P. Os grupos Alfa e a adesão ao Regime Remuneratório Experimental. Rev Port Clin Geral 2005; 21:45-59.

16. Portugal. Decreto-Lei $\mathrm{n}^{\circ} 117 / 98$, de 5 de Maio de 1998. Diário da República 1998; 5 maio.

17. Portugal. Decreto-Lei $n^{\circ} 157 / 99$, de 10 de maio de 1999. Centros de saúde de terceira geração. Diário da República 1999; 10 maio.

18. Pisco L. Reforma da Atenção Primária em Portugal em duplo movimento: unidades assistenciais autónomas de saúde familiar e gestão em agrupamentos de Centros de Saúde. Cien Saude Colet [Internet]. 2011 Jun [acessado 2019 Set 14]; 16(6):2841-2852. Disponível em: http://www.scielo.br/scielo.php?script=sci_arttext\&pid=S1413-81232011000600022\&lng=pt
19. Portugal. Resolução do Conselho de Ministros no 157/2005, de 22 de setembro de 2005. Diário da República 2005; 22 set.

20. Portugal. Resolução do Conselho de Ministros $n^{\circ}$ 157/2005, de 12 de outubro de 2005. Linhas de Acção Prioritária para o Desenvolvimento dos Cuidados de Saúde Primários. Missão para os Cuidados de Saúde Primários. Diário da República 2005; 12 out.

21. Portugal. Serviço Nacional de Saúde (SNS). Mais utentes com médico atribuído. Lisboa, 19 de junho de 2019. [acessado 2019 Set 18]. Disponível em: https:// www.sns.gov.pt/noticias/2019/06/19/mais-utentescom-medico-atribuido/

22. Soranz D, Pinto LF, Penna GO. Eixos e a Reforma dos Cuidados em Atenção Primária em Saúde (RCAPS) na cidade do Rio de Janeiro, Brasil. Cien Saude Colet [Internet]. 2016 Maio [acessado 2019 Set 14]; 21(5):1327-1338. Disponível em: http://www.scielo.br/scielo.php?script=sci_arttext\&pid=S1413-81232016000501327\&lng $=p t$

23. Soranz D, Pisco LAC. Reforma dos Cuidados Primários em Saúde na cidade de Lisboa e Rio de Janeiro: contexto, estratégias, resultados, aprendizagem, desafios. Cien Saude Colet [Internet]. 2017 Mar [acessado 2019 Set 14]; 22(3):679-686. Disponível em: http://www.scielo.br/scielo.php?script=sci_arttext\&pid $=$ S1413-81232017002300679\&lng $=p t$

24. The Lancet. The Astana Declaration: the future of primary health care? Lancet 2018; 392(10156):1369.

25. Lancet. Investment in health is key to boosting wealth. Lancet 393(10176):1072.

26. World Health Organization (WHO), United Nations Children's Fund (UNICEF). Global conference on primary health care: from Alma-Ata towards universal health coverage and the Sustainable Development Goals, Astana, Kazakhstan, 25 and 26 October 2018. Geneva: WHO; 2018.

27. Pizarro M. Celebrar o SNS é fazer o que ainda não foi feito. Portugal. Jornal Expresso online 2019; 13 set. p. 1.

28. Temido M. Ministra da Saúde. Discurso de encerramento das comemorações dos 40 anos do SNS, que decorreram no Centro Hospitalar e Universitário de Coimbra. 17 de setembro 2019. Coimbra: Centro Hospitalar e Universitário de Coimbra; 2019.

29. Portugal. Lei no 95, de 4 de setembro de 2019. Lei de Bases da Saúde de 2019. Diário da República 2019; 4 set.

Artigo apresentado em 23/10/2019

Aprovado em 24/10/2019

Versão final apresentada em 26/10/2019 